\title{
SOP 09: Statistical Design and Analysis
}

\section{Background}

Statistical design and analysis of a CESAR study on the efficacy and/or safety of an anticancer drug should comply with ICC-GCP guidelines on statistical principles for clinical trials. Therefore, when not stated otherwise explicitly, the ICH-E9 guideline [1] and its rules will be applied to a CESAR study. The subsequent paragraphs will give specific guidance to special issues of CESAR trials. The respective ICH-E9 guideline will be cited below where applicable in its issue from February 1998.

The biometric center (BC) of CESAR is responsible for contributing biostatistical expertise during the planning and the analysis stage of a CESAR study. Its mission is to support and to promote the scientific advancement of anticancer drug research.

The tasks of the BC are guided by the primary objective(s) of a study and the goal of accomplishing a successful clinical trial. Therefore, the BC contributes especially to

- The statistical design of the study and the determination of the minimal number of patients required,

- The standardized documentation and the recording of the study data in accordance with the trial protocol,

- The computerized storage, processing, and statistical analysis of the data,

- The interpretation and reporting of the trial results in cooperation with the sponsor, the monitor, the Principal Investigator, and the clinical investigators.

Specifically the BC is committed

- For phase I studies to the

- Determination of appropriate dose ranges and identification of the maximal tolerable dose (MTD),

- Drug safety analysis;

- For phase II studies to the

- Determination of the anticancer activity of the trial drug in terms of objective (measurable) criteria,

- Drug safety analysis;
- For phase III studies to the

- Determination of a treatment effect in comparative randomized clinical trials on anti-cancer drug efficacy,

- Drug safety analysis.

\section{Statistical Issues of the Trial Protocol}

The BC shall assume responsibility for the statistical sections of the trial protocol and play an active part in the protocol's formulation or provide the expertise necessary for its completion.

The tasks involve at least the following items:

- Ensuring the adequate definition of the endpoint(s) and the clinically important effects as a prerequisite for establishing the statistical design,

- Specification of statistical hypotheses and error probabilities in phase II-III studies,

- Mode of randomization and blinding as well as conditions for breaking the code,

- Calculation of the minimal number of patients to be enrolled in the study,

- Rule(s) for stopping patient accrual,

- Statistical analysis plan.

The BC will review the trial protocol and the trial's Case Report Forms (CRFs) with regard to at least the following aspects:

- Statistical evaluability of the study endpoints such as survival time, time to progression, tumor response rate, duration of remission or incidence of adverse event,

- Definition of the study population (inclusion/exclusion criteria) and analysis sets (e.g. for the intent-to-treat analysis and the per protocol analysis),

- CRFs and the logistics of the data flow,

- Patient evaluability criteria.

\section{Statistical Study Design}

Phase I Study: The trial protocol will specify a sequential dose escalation scheme (e.g. a choice of doses according to 
the modified Fibonacci scheme) and implement a stopping rule for each dose, e.g. the traditional escalation rule or a modified continual reassessment rule [2, 3].

Phase II Study: The selection of a treatment plan must allow for early termination of a regimen that turns out to be ineffective, e.g., implementing a plan from the class of the optimal two-stage designs. The plan should control the probability of inadvertently rejecting an effective agent/regimen and the probability of falsely accepting an ineffective agent [4].

Phase III Study: The trial protocol has to specify the type of statistical hypotheses to be tested for the efficacy statement (e.g. superiority, equivalence, noninferiority, [1, section 3.3]) and the statistical error probabilities which are the error of the 1st kind ( $\alpha$-error, significance level) and the error of the 2 nd kind ( $\beta$-error) which defines the power of the trial. If more than one endpoint is considered as primary (e.g. survival and toxicity in combination), specific designs have to be implemented to prevent erroneous results arising from multiple testing.

Were applicable the design should identify primary and secondary endpoints [1, section 2.2.2].

\section{Patient Registration and Control of the Study}

Phase I Study: The BC will keep a list of the patients entered on the study. This list contains at least the following information:

- Study identification,

- Institution/investigator identification,

- Patient identification by initials, date of birth, and running number,

- Date of registration at sponsor's institution or at the $\mathrm{BC}$ as defined in the study protocol,

- Target dose on admission.

Phase II Study: The BC will keep a list of the patients entered on the study, in particular to ensure that the statistical rule for stopping patient accrual is adhered to. This list uses the patient data submitted to the $\mathrm{BC}$ for the eligibility check (see 'SOP 7: Data Flow, Monitoring, and Archiving') and contains at least the following information:

- Study identification,

- Institution/investigator identification,

- Patient identification by initials, date of birth, and running number,

- Date of registration at sponsor's institution.

The BC will compare the number of recruited cases with the target sample size of the study at regular intervals. Once the total number of patients or a relevant proportion of the target number for the continuation of the study have been enrolled, the $\mathrm{BC}$ will notify the sponsor and the Coordinating Investigator $(\mathrm{CI})$. The $\mathrm{BC}$, the sponsor, and the $\mathrm{CI}$ will then jointly decide on the continuation of patient accrual.
When a two-stage design is used and the initial accrual goal of evaluable patients has been met, the $\mathrm{BC}$ will instruct the CI and the sponsor to promptly determine the result of the relevant study endpoint (typically tumor response) for each evaluable subject. This requires temporary closure of patient accrual and will be decided on in concert with the sponsor and the CI.

When a randomized phase I/II study is performed, the BC will be responsible for the randomization of patients to the different treatment arms, unless otherwise specified in the study protocol.

Phase III Study: The BC will keep a list of the patients entered to ensure that the statistical rule for stopping patient accrual is adhered to. This list uses the patient data submitted to the BC for the eligibility check (see 'SOP 7: Data Flow, Monitoring, and Archiving') and contains at least the following information:

- Study identification,

- Institution/investigator identification,

- Patient identification by initials, date of birth, and running number,

- Date of registration at sponsor's institution.

The BC will compare the number of evaluable cases with the target sample size of the study at regular intervals for check-up with the interim analysis plan of the trial and will notify the $\mathrm{CI}$ and the sponsor when decisions have to be made.

The $\mathrm{BC}$ is responsible for the randomization of patients into the different treatment arms (randomization procedure, randomization list, recording of randomization and information of CI and the sponsor). The rules for implementing and performing the randomization will be those of ICH E9, 2.3.2 [1]. Unless otherwise specified in the study protocol the $\mathrm{BC}$ will be responsible for ensuring blinding [see also 1, section 2.3.1].

Intergroup Studies: In intergroup studies, the $\mathrm{BC}$ of $\mathrm{CE}$ SAR will be involved in the biostatistical analysis at least as a reviewer. Details have to be agreed on by the sponsor and the $\mathrm{BC}$ and must be laid down in writing.

\section{Statistical Analysis}

\section{Statistical Documentation of Study Data}

All data, findings, and other observations to be recorded as stipulated by the study protocol will be computerized. Data entry into the computer is based on the CRFs and, where appropriate, its attachments. As a rule, all study data reported on the CRFs will be included in the statistical analysis. Comments will be entered as such only if they are explicitly requested by the study protocol. The presence/absence of comments in free format on individual CRFs will be identified by a separate entry. The data will be handled by the $\mathrm{BC}$ according to procedures which comply with ICH-GCP guidelines, including quality assurance, 
e.g. by implementation of a double entry system. Details deviating from the procedures specified below have to be documented in study-specific procedures.

\section{Computer Entry System and Data Management}

The BC uses a computer entry system and data management software the use, function, description, and documentation of which are documented in detail in the BC's own SOP.

\section{Quality Assurance}

All data are entered from the original (firstst page/white page) of the CRFs (see 'SOP 7: Data Flow, Monitoring, and Archiving').

Corrections and Missing Data: If a form contains contradictory or unclear entries, it will be returned to the responsible investigator via the study monitor while a copy is kept on file at the BC. Query handling follows the procedures defined in 'SOP 7: Data Flow, Monitoring, and Archiving'. The guidelines for correcting computerized data are specified in the BC's SOP.

After each updating of the database, the BC makes a list of CRFs that are still missing or have not been submitted in time and forwards it to the monitor of the sponsor.

Laboratory Values: As a rule, laboratory values are entered using the units given on the CRFs. For each laboratory of an institution which performs laboratory tests for a study patient, the form 'Validated Laboratory Normal Ranges' has to be completed, sent to the $\mathrm{BC}$ and to the monitor of the sponsor, and kept in file at the BC. If other units than those required on the CRFs are used, that different unit has to be given on the CRFs and on 'Validated Laboratory Normal Ranges' together with the conversion factor used. Unless otherwise specified, the monitor of sponsor is responsible for the correct conversion and the correct entry on the CRF in the units prespecified by the CRF. If laboratory tests for an individual patient are performed by different laboratories, the name of the laboratory has to be given for each laboratory value recorded on the CRF.

\section{Patient Eligibility}

All patients who are registered by the clinical investigators to be included into the study are considered as of being recruited (enrolled) for the study. This is documented by the application with the monitor of the sponsor or by the application with the $\mathrm{BC}$ (e.g. in the case of the request for a randomization in a phase III trial). Special application forms should be used for patient registration. All patients who have been successfully checked for the correctness of the in/exclusion criteria and who have been confirmed by the monitor of the sponsor or the $\mathrm{BC}$ as of being included into the study are eligible for the study. These patients represent the intent-to-treat population of a phase II or phase III study. The Full Analysis Set and the Per Protocol Set of patients is defined within this population of patients eligible for the study according to the ICH-GCP [1, section 5.2].

\section{Patient Evaluability}

This section defines guidelines for the assessment of evaluability of study patients for biostatistical analysis and aims at implementing measures to reduce selection bias. A distinction is made between complete and partial evaluability (e.g. patient not evaluable for response but evaluable for safety).

- As a rule, the data of each patient eligible for a study (see 'Patient Eligibility') are checked for evaluability on the basis of the criteria specified in the study protocol.

- The evaluability check will typically take into account the following elements of the study:

- Inclusion/exclusion criteria,

- Time schedule of patient presentation at the institution, data recording and measurement,

- Compliance with the treatment plan,

- Completeness and quality of the documentation,

- Concomitant disease(s),

- Concomitant treatment(s).

- The decision to fully or partly exclude a patient from an analysis will be made by the $\mathrm{BC}$ in concert with the sponsor and the CI.

- The reasons for complete or partial exclusion from analysis must be specified in a written report on a case-bycase basis, which should be included in the Biometrical Report.

- Patient data that are excluded from statistical analysis shall be listed separately in the Biometrical Report.

- Evaluability for response to treatment in phase II studies. If not specified otherwise in the study protocol all patients who were eligible for a phase II study are evaluable for response if they received the trial drug for at least 1 cycle or at least for 4 weeks (treatment limit) and if the documentation is adequate for the assessment of response.

- Evaluability for safety. All patients who were eligible for a study are evaluable for safety if they received the trial drug at least once and if the documentation is adequate.

- The statistical analysis of a comparative trial, in particular a phase III trial, has to include always a confirmative statistical analysis for intent-to-treat, e.g. by an evaluation of the full analysis set according to ICH-GCP [1, section 5.2].

\section{Statistical Analysis}

Statistical analysis is performed according to the statistical analysis plan specified in the study protocol. Some impor- 
tant aspects regarding the analysis of phase I-III studies are regulated in the subsequent sections.

Survival: Overall survival will be evaluated statistically by applying the methods for censored survival times. This includes the descriptive analysis using Kaplan-Meier estimates of the survival curve, the log-rank test for the comparisons of survival curves, and the use of the Cox proportional hazard regression model for adjusting the treatment comparison for the effect of confounding prognostic factors. Risk ratios will be calculated with their $95 \%$ confidence intervals on the basis of the results of the Cox regression and where it is possible also based on the numbers of observed $(\mathrm{O})$ and expected (E) events (for the basic methodology see [5]). For testing a noninferiority alternative of survival the method described by Wellek [6] is used.

Response: The endpoint tumor response is assessed through the rate of objective responses. It is defined as the quotient of the number of patients evaluated as complete or partial remissions (CR or PR) and the number of patients evaluable for response. The variability of the response rate is evaluated by calculating the $95 \%$ confidence interval. For study populations of up to $n=100$ cases, this calculation uses the exact limits from Geigy's tables [7].

Eligible patients lost during the first 4 weeks or the first treatment cycle are listed separately in the response tables. The reasons for the loss of a patient or discontinuation of treatment in individual cases must be specified. At least the following cases shall be distinguished:

- Early death within first cycle / 4 weeks,

- Early progression within first cycle / 4 weeks,

- Lost to follow-up,

- Lack of evaluation/missing documentation,

- Treatment refused by patient,

- Treatment discontinued by investigator.

In addition, an intent-to-treat response rate is calculated, which includes all eligible patients.

Unless otherwise stated, the response evaluation is based on the RECIST and/or the WHO criteria and the assessment of the tumor lesions of a patient is within a prespecified window of treatment cycles designated for response evaluation (e.g. from the end of treatment cycle 2 until the end of treatment cycle 4).

Further analyses, e.g. response including the status of 'persistent status quo' or 'no change' [see 8]; 'SOP 3: Preparation and Structure of Trial Protocols') or so-called 'best response' based on a maximum interval of individual patient follow-up, are considered as secondary response evaluation, unless specified differently in the study protocol. Safety: All eligible patients are evaluable for safety if they have received the investigational drug at least once and documentation is adequate. For analysis of an individual safety parameter, an initial value prior to the start of treatment must be available for comparison.
Cases excluded from safety analysis are reported separately with reasons for their exclusion. Partial exclusion of patients is permissible (e.g. exclusion from the analysis of single safety parameters) without affecting that patient's general evaluabilty. [see also 1, section 6.1-6.5].

Interim Reports: An interim analysis is any analysis intended to compare treatment arms with respect to efficacy or safety at any time prior to formal completion of a trial. Because the number, methods and consequences of these comparisons affect the interpretation of the trial, all interim analyses should be carefully planned in advance and described in the protocol.

Not every oncological trial requires interim analyses or implementation of group-sequential design. A clinical trial on the efficacy of a treatment assessed through overall survival as endpoint may recruit the majority of patients much faster than the data are available to perform an interim analysis with a proportion of the total population planned for this study (recruitment overruns the interim analysis). When an interim analysis is planned with the intention of deciding whether or not to terminate a trial, this is usually accomplished by the use of a group-sequential design which employs statistical monitoring schemes [1, section 4.5]. Among others, the following measures should be taken:

- The protocol should describe the schedule of interim analyses or, at least, the considerations which will govern its generation.

- The execution of an interim analysis should be a completely confidential process, at least, with respect to the endpoint(s) or any other endpoint which could affect the results of the primary endpoint(s).

Interim reports regarding the primary endpoint(s) of all open studies are presented at the regular meetings of Phase I, Phase II or Phase III Working Groups of CESAR Such reports contain the following information:

- Administrative data on patient accrual rate, patients off study, eligibility, and evaluability,

- Summary of patient characteristics,

- Statement on the status of a study without giving results on the endpoints,

- Summary of the most important safety parameters from the CTC checklist.

A summary statement on study endpoints (e.g. overall survival, time to progression, response rate) is only allowed if this statement is ensured not to interfere with the ongoing patient accrual and to imply any bias to the study result. It is not allowed to unblind the results of any study endpoints in a randomized phase III trial.

Final Analysis: The final analysis is done on the basis of the complete documentation of all cases and serves as a basis for the study report and for the publication of the results. It will be done in accordance with the statistical analysis plan outlined in the trial protocol. The analysis includes at least the following variables:
Standard Operating Procedures for Clinical Trials of the CESAR Central European Society for Anticancer Drug Research - EWIV 
- Patient characteristics

- Age,

- Sex,

- Performance status,

- Stage of the disease,

- Localization of metastases,

- Prior treatment,

- Concomitant disease(s);

- Treatment-related data

- Duration of treatment,

- Cumulative dose,

- Reason for discontinuation of treatment,

- Dose intensity;

- Study endpoints

- Survival,

- Response,

- Toxicity (according to CTC criteria, including baseline values and extreme values).

\section{Biometric Report/Statistical Report}

The final analysis (cf. 'Statistical Analysis') serves as a basis for the preparation of the Biometric Report/Statistical Report of the results of the statistical analyses performed by the BC. This report will be sent to the sponsor and the CI. The report provides a detailed description of the results of the study including their biostatistical interpretation. The guidelines for writing the Statistical Report are defined in the data center's SOP 'Statistical Report'.

The biometric report/statistical report gives a detailed description of the study population and will comply with the CONSORT Statement [9]. It comprises at least the following items:

- Registered patients,

- Eligible patients,

- Protocol violations including a detailed description,

- Evaluable cases,

- Patients evaluable for survival times,

- Patients evaluable for response,

- Patients evaluable for safety.

The biometric report/statistical report covers at least the following areas:

- Patient characteristics,

- Results of treatment,

- Safety.

Part of the results can be summarized in tables. The report must be sent to the CI within 12 weeks of receipt of the last patient documentation.

\section{Documentation and Archiving of Study Data}

Data Listing: The data listing is a reproduction of the CRFs. As a rule, a full listing is compiled after completion of the (final) analyses for a given study. This listing will be made available to the $\mathrm{CI}$ and the sponsor.

Data Exchange: All exchange of data within the $\mathrm{BC}$ and between the $\mathrm{BC}$ and the sponsor will be validated. The procedures are defined in the SOP 'Data Transfer between $\mathrm{BC}$ and Sponsor' established by the BC.

Confidentiality, Security and Archiving of Data: The study data are saved on appropriate media (e.g. on a file server with a routinely implemented data security backup) on a daily basis after completion of data entry. Backups of the data are made at regular intervals, at least every 4 weeks. Data used for any interim and final analysis will be stored on a diskette or a CD. Protection of the data from unauthorized users is ensured by a password. The backup storage files are kept in the study files together with the CRFs, protected from any unauthorized access.

Hardware Configuration and Software Used at the BC: Electronic data processing for analyses conducted by the $\mathrm{BC}$ of CESAR is performed in the Biostatistics Unit at the Deutsches Krebsforschungszentrum (DKFZ, German Cancer Research Center). The computer systems available for data management and analysis comprise PCs in NOVELL net of the data processing center of the DKFZ and the decentralized networks of the Biostatistics Unit (SUN Cluster).

As a rule, statistical analyses are performed using SAS [9] or Splus in its most recent version. Additional statistical tasks can be done with validated software of the Biostatistics Unit of the DKFZ. Detailed information on the currently used computer systems, the versions of SAS and additional software is provided in the BC's SOP, which also defines the validation of nonstandard software.

\section{References}

1 ICH E9: Note for Guidance on Statistical Principles for Clinical Trials, CPMP/ICH/363/96, 5 February 1998.

2 Edler L: Overview of phase I trials; in Crowley J (ed): Handbook of Statistics in Clinical Oncology. New York, Marcel Dekker, 2001,pp 1-34.

3 Hanauske AR, Edler L: New clinical trial designs for phase I studies in hematology and oncology. Principles and practice of the continual reassessment model. Onkologie 1998;19:404-409.

4 Edler L: Statistical requirements of phase I studies. Onkologie 1990;13: 90-95.

5 Kalbfleisch JD, Prentice RA: Statistical Analysis of Failure Time Data. New York, Wiley, 1980.

6 Wellek S: A log-rank test for equivalence of two survivor functions. Biometrics 1993;49:877-881.

7 Wissenschaftliche Tabellen Geigy, Teilband Statistik, 8. Auflage. Basel, Geigy, 1980.

8 Edler L, Flechtner H: Remission in Phase-II und Phase-III Studien: Kriterien und Voraussetzungen. Onkologie 1987;10:330-339.

9 Begg C, Cho M, Eastwood S, et al: Improving the quality of reporting of randomized controlled trials. The Consort Statement. JAMA 1996;276:637-639.

10 SAS Institute Inc: SAS Procedures Guide, Version 6, 3rd ed. Cary, SAS Institute Inc., 1990 